\title{
REFLEXÃO ACERCA dA POLÍTICA DO MINISTÉRIO DA SAÚdE BRASILEIRO PARA A ATENÇÃo AOS USUÁRios de ÁlCOOL E OUTRAS DROGAS SOB A ÓPTICA DA SoCIOLOGIA DAS AUSÊNCIAS E DAS EMERGÊNCIAS
}

\author{
Maria Odete Pereira ${ }^{1}$; Divane Vargas $^{2}$; \\ Márcia Aparecida Ferreira de Oliveira ${ }^{3}$
}

\begin{abstract}
Em 2003 foi implantada a Política Nacional do Ministério da Saúde do Brasil para a atenção aos usuários de álcool e outras drogas. Estudo interpretativo e documental que objetivou refletir acerca da referida Política, sob o referencial teórico da Sociologia das ausências e das emergências. A Política emergiu dos movimentos sociais que, por mais de duas décadas, reivindicaram formas inclusivas de cuidado, pois o modelo hospitalocêntrico era o de exclusão. As origens contra-hegemônicas conferem legitimidade à Política, que se propõe à reabilitação psicossocial e à emancipação social das pessoas com existênciasofrimento. As ações praticadas pelas equipes de saúde mental nos territórios é que garantirão o seu sucesso.
\end{abstract}

Descritores: Políticas Públicas; Alcoolismo; Toxicologia; Gestão em Saúde; Ciências Sociais; Enfermagem.

\footnotetext{
${ }^{1}$ Enfermeira, Doutor em Enfermagem, Professor, Faculdades Integradas Teresa D'Ávila, Lorena, SP, Brasil. E-mail: m.odetepereira@gmail.com

2 Enfermeiro, Doutor em Enfermagem, Professor Doutor, Escola de Enfermagem, Universidade de São Paulo, SP, Brasil. E-mail: divanidevargas@gmail.com.

${ }^{3}$ Enfermeira, Livre Docente, Professor Associado, Escola de Enfermagem, Universidade de São Paulo, SP, Brasil. E-mail: marciaap@usp.br.
} 


\section{Reflection on the policy of the Brazilian Ministry of Health for THE CARE OF ALCOHOL AND OTHER DRUGS USERS UNDER THE VIEW OF THE Sociology of AbSences ANd Emergencies}

IN 2003, the National Policy of the Brazilian Ministry of Health was implemented to care for users of alcohol and other drugs. This is a interpretative and documental research with the objective of reflecting on the refereed Policy, under the theoretical reference of the Sociology of Absences and Emergencies. The Policy came from the social movements that for more than two decades have demanded inclusive forms of care, since the centrichospital model was exclusion. The against-hegemonic origins give the Policy legitimacy, which proposes the psychosocial rehabilitation and the social emancipation of people with existence-suffering. The action practiced by the mental healthcare teams in the territories is what will ensure its success.

Descriptors: Public Policies; Alcoholism; Toxicology; Health Management; Social Sciences; Nursing.

\section{Ponderación ACERCA de la POLÍTiCA Del Ministerio de LA SALUd} BRASILEÑO PARA LA ATENCIÓN A LOS USUARIOS DE ALCOHOL Y OTRAS DROGAS BAJO LA ÓPTICA DE LA SOCIOLOGÍA DE LAS AUSENCIAS Y DE LAS EMERGENCIAS

En 2003 fue implantada la Política Nacional del Ministerio de la Salud de Brasil para la atención a los usuarios de alcohol y otras drogas. Estudio interpretativo y basado en documentos que objetivó reflejar acerca de la referida Política, bajo la referencia teorética de la Sociología de las ausencias y de las emergencias. La Política emergió de los movimientos sociales que, por más de dos décadas, reivindicaron maneras inclusivas de cuidado, pues el modelo hospitalocéntrico era lo de exclusión. Los orígenes contrahegemónicos confieren legitimidad a la Política, que se propone a la rehabilitación psicosocial y a la emancipación social de las personas con existencia-sufrimiento. Las acciones practicadas por los equipos de salud mental en los territorios es que garantizarán su éxito.

Descriptores: Políticas Públicas; Alcoholismo; Toxicología; Gestión en Salud; Ciencias Sociale; Enfemería.

Introdução

O uso nocivo de álcool e outras drogas é considerado desordem multifatorial. Infelizmente, em muitas sociedades, ainda não é considerado problema de saúde pública e muitas pessoas sofrem, pois são estigmatizadas e não têm acesso a tratamento e reabilitação psicossocial ${ }^{(1)}$.

O álcool é uma substância presente no cotidiano de praticamente todas as pessoas. O seu uso é considerado comum na nossa sociedade, estando relacionado a festas e comemorações, e a questões valorizadas socialmente, como prazer, liberdade e lazer. Beber é socialmente aprovado entre diversos grupos sociais, o que dificulta o estabelecimento de limites entre o consumo recreativo e a 
dependência ${ }^{(2)}$.

Estima-se que 205 milhões de pessoas utilizem drogas ilícitas, incluindo os 25 milhões que delas são dependentes. Isso se constitui um problema de saúde pública; afeta o desenvolvimento socioeconômico e gera demandas relacionadas à segurança, nos países industrializados e em desenvolvimento $^{(1)}$. Assim, as consequências negativas dessa condição têm repercussões familiares, sociais, sobre a saúde, além do significativo impacto econômico ${ }^{(3)}$.

O uso contínuo das drogas psicoativas pode impactar indicadores epidemiológicos como: mercado de consumo de bebidas alcoólicas, acidentes de trânsito, violência e criminalidade e internações hospitalares, além de comportamentos antissociais, ineficiência em trabalhos que exijam a força e dificuldades para os familiares dos usuários $^{(4-5)}$.

O consumo nocivo de álcool gera custo social significativamente maior que o provocado pelo tabaco e outras drogas. Estima-se que o Brasil gaste, anualmente, $7,3 \%$ do Produto Interno Bruto (PIB) com tratamento e assistência social relacionados ao seu uso ${ }^{(4-6)}$.

A amplitude e a gravidade dos problemas referidos acima exigem que os órgãos governamentais de todos os países adotem políticas e estratégias que diminuam o uso de drogas pela população em geral, bem como evitem as consequências da utilização nociva dessas substâncias. No entanto, não se pode traçar uma política com apenas um objetivo: o da abstinência, pois trabalhar dessa forma, em saúde, significa trabalhar com um entendimento muito estreito acerca da questão(7).

As políticas de saúde para as questões relacionadas ao consumo nocivo de álcool e outras drogas apresentam diferentes conformações e objetivos, e este artigo objetiva analisar a Política Nacional do Ministério da Saúde brasileiro para a atenção integral aos usuários de álcool e outras drogas ${ }^{(8)}$, na perspectiva de um referencial teórico sociológico, uma vez que não há estudos similares publicados.

A pergunta que aqui se faz é se a Política em questão atende as demandas dos usuários de álcool e outras drogas. Ademais, os autores querem verificar se ela coaduna com as demandas dos usuários de substâncias psicoativas.

\section{Métodos}

O estudo qualitativo, interpretativo e documental constitui parte da fundamentação teórica da tese de doutoramento de uma das autoras.

A pesquisa documental permite o exame de materiais que ainda não foram submetidos a um tratamento analítico, ou que possam ser reexaminados para nova interpretação ou complementação. Pode servir de base para outros estudos qualitativos, além de possibilitar que a criatividade do pesquisador dirija a investigação sob diferentes perspectivas ${ }^{(8)}$.

Para a análise da Política Nacional brasileira do Ministério da Saúde, em atenção aos usuários de álcool e outras drogas, a fim de verificar se a mesma atende às necessidades dos usuários, será utilizado o referencial teórico da Sociologia das ausências e das emergências, construtos de uma teoria crítica, denominado Paradigma Emergente, que preconiza "um conhecimento prudente para uma vida decente"(10). A construção teórica é de oposição à racionalidade moderna e, por isso, denominada crítica.

$\mathrm{Na}$ óptica da Sociologia das ausências, o conhecimento, para ser profícuo, deve permitir ser aplicado no campo social e promover transformações na vida das pessoas, a fim de que tenham uma vida decente.

A Sociologia das ausências é proposta como procedimento para ampliação do mundo e dilatação do presente. Trata-se de lógica "que visa demonstrar que o que não existe é, na verdade, ativamente produzido como não existente, isto é, como uma alternativa não credível ao que existe" ${ }^{(10)}$.

O objetivo da Sociologia das ausências é o de transformar objetos impossíveis em possíveis e com base neles transformar ausências em presenças, centrando-se nos fragmentos das experiências sociais não socializadas pela racionalidade de totalidade metonímica. Nesse sentido, todo esforço deve ser empreendido no sentido de ampliar as experiências de vida do presente, dos usuários de álcool e outras drogas, de forma a contrair o futuro, aproximando-o ${ }^{(10)}$.

A construção teórica defende a tese de que uma racionalidade cosmopolita se propõe a expandir o presente e contrair o futuro, pois só assim se pode criar o espaçotempo necessário para se conhecer e valorizar a inesgotável experiência social que acontece hoje no mundo, evitando o imenso desperdício que se tem dessas experiências. Para expandir o presente, propõe-se uma Sociologia das ausências e, para contrair o futuro, uma Sociologia das emergências $^{(10)}$.

O papel da Sociologia das emergências é o de substituir o vazio do futuro, segundo o tempo linear (um vazio que tanto é tudo como é nada), por um futuro de possibilidades plurais e possíveis, portanto, concretas. Isso se dá com as atividades de cuidado que se tem no presente $^{(10)}$.

\section{Resultados}

A atual Política Nacional do Ministério da Saúde brasileiro para a atenção integral aos usuários de álcool e outras drogas, que está a ser analisada, foi instituída por meio da Portaria $n^{\circ} 2.197 / \mathrm{GM}$, em 14 de outubro de 2004. A análise da Política permitiu verificar no que ela se coaduna com as demandas dos usuários de substâncias psicoativas.

No Brasil, a exclusão social e ausência de cuidados marcaram a existência dos portadores de transtornos mentais, usuários e dependentes de álcool e outras drogas, pois as intervenções à saúde dos usuários e dependentes de álcool e outras drogas no País, ao longo da história, se resumiram em iniciativas de atenção de caráter total, fechado, tendo a abstinência como único objetivo a ser alcançado $^{(8)}$.

A Lei 10.216, de 6 de abril de 2001, que se 
constitui no marco histórico para a Reforma Psiquiátrica Brasileira, ratificou a Lei 8080/90, que estabelece as diretrizes do Sistema Único de Saúde, garantindo, a todos os indivíduos usuários e dependentes de álcool e outras drogas, a universalidade de acesso à assistência integralmente, priorizando a descentralização dos serviços de atendimento, determinando que os mesmos devam ser estruturados na comunidade, próximos do convívio social dos usuários. Assim, as redes assistenciais devem centrarse nas desigualdades existentes, ajustando suas ações às demandas da população, para atender de forma equânime e democrática ${ }^{(8,11)}$.

Pautando-se nas concepções da Reforma Psiquiátrica, iniciou-se a construção da rede assistencial baseada em dispositivos extra-hospitalares de atenção psicossocial, localizada no território e articulada com outros setores sociais. Rede essa que objetiva a reinserção social dos sujeitos que vivenciam uma existência-sofrimento ou que já desenvolveram problemas psíquicos, decorrentes do uso abusivo de álcool e outras drogas ${ }^{(12)}$.

No âmbito do Sistema Único de Saúde, a Portaria GM/816, de 30 de abril de 2002, instituiu o Programa Nacional de Ação Comunitária Integrada aos Usuários de Álcool e outras Drogas, levando em consideração a multiplicidade de níveis organizacionais das redes assistenciais localizadas nos Estados e Distrito Federal, os diferentes perfis populacionais existentes no País e a variabilidade de incidência dos transtornos decorrentes do uso abusivo e/ou dependência de álcool e outras drogas.

Com isso, propôs-se a implantação dos Centros de Atenção Psicossocial 'Álcool e Drogas - CAPSad, com o objetivo de melhorar a assistência em saúde mental e abrigar, nos projetos terapêuticos, práticas de cuidados que contemplem a flexibilidade e a abrangência possíveis às demandas da população atendida, sob perspectiva estratégica de redução de danos sociais e à saúde ${ }^{(8)}$.

Considerando os avanços na acessibilidade, cobertura e qualidade de atendimento em saúde mental, o Ministério da Saúde instituiu, por meio da Portaria GM 678, de 2006, a Estratégia Nacional de Avaliação, Monitoramento, Supervisão e Apoio Técnico aos CAPS e outros serviços da rede pública de saúde mental do SUS. A coordenação de Saúde Mental do Ministério da Saúde brasileiro divulgou, em junho de 2010, que o Brasil conta com 242 CAPSad no território nacional ${ }^{(8)}$.

Percebe-se que o uso do álcool e outras drogas é tema transversal a outras áreas da saúde, pois envolve a justiça, a educação, o bem-estar social e o desenvolvimento. Portanto, faz-se necessária uma série de articulações intersetoriais com vários setores da sociedade civil, como movimentos sindicais, universidades e organizações comunitárias. Essas articulações são essenciais para a elaboração de planos estratégicos dos Estados e municípios, de forma a ampliar as ações dirigidas às populações de difícil acesso, além de serem instrumentos essenciais para a promoção de direitos e controle social ${ }^{(8)}$.

Em 2005, através da Portaria GM 1612 regulamentaram-se os mecanismos de financiamento dos leitos para álcool e outras drogas nos hospitais gerais -
HG, objetivando que os usuários de substâncias químicas fossem internados em leitos de hospitais gerais e não mais em hospitais psiquiátricos. No entanto, o número de leitos em HG ainda é insuficiente para atender à demanda ${ }^{(11)}$.

Segundo dados do Ministério da Saúde, de junho de 2010, o Brasil dispõe de 2.568 leitos em 415 HG. Com isso, muitos usuários, em situações que exigem internações, ainda são internados em leitos para desintoxicação de álcool e outras drogas, em hospitais psiquiátricos, clínicas privadas ou em comunidades terapêuticas, que, muitas vezes possuem condições de hotelaria inadequadas.

Implantou-se, em 2005, o Pacto Nacional para Redução de Acidentes e Violência Associados ao Consumo Prejudicial de Álcool, sob a coordenação dos Ministérios da Saúde e Justiça, Secretaria Nacional de Políticas sobre Drogas - Senad e Frente Nacional de Prefeitos. No mesmo ano, a Portaria 1.059, de 2005, regulamentou os incentivos financeiros para os CAPSad que desenvolvam ações de redução de danos. Para os municípios que tenham apenas CAPS I, autorizou-se que cobrem do SUS pelos atendimentos às pessoas com transtornos decorrentes do uso de álcool e outras drogas, por meio da Portaria SAS 384, de $2005^{(11)}$.

No Programa, o controle de entorpecentes e substâncias que produzem dependência física ou psíquica é regulado pela Anvisa - Agência Nacional de Vigilância Sanitária, de acordo com a Portaria SVS/MS n³44/98, competências executadas pelo Ministério da Saúde, sob amparo do artigo $6^{\circ}$ da Lei ${ }^{\circ} 6368 / 76^{(6)}$. Da mesma forma, os serviços residenciais terapêuticos, instituídos para possibilitar a reabilitação psicossocial das pessoas com dependência ao álcool e outras drogas, são regulados por meio da Resolução da Diretoria Colegiada de nº101/2001, de maio de 2001. Com essas atribuições, a Anvisa se inclui entre os atores que aceitaram o desafio do enfrentamento das questões referentes ao álcool e outras drogas, com seus resultantes psíquicos, sociais e econômicos - um dos maiores problemas da sociedade contemporânea ${ }^{(8)}$.

O Ministério da Saúde, atualmente, está investindo esforços na incorporação de ações de redução de danos por outros programas do SUS como nos Programas de Agentes Comunitários e da Saúde da Família. No entanto, para o Ministério, a redução de danos deve ser a lógica empregada como referencial para as ações políticas, educativas, terapêuticas e preventivas, em todos os níveis. A Portaria GM 1028, de 2005, regulamenta as ações que visam a redução de danos sociais e à saúde, retirando da relativa clandestinidade institucional essa valiosa estratégia de abordagem dos problemas associados ao consumo de álcool e outras drogas ${ }^{(8)}$.

Ações como a restrição ao uso de cigarros e outros produtos derivados do tabaco, em recintos coletivos, são estratégias de redução de danos, que respeitam a opção individual de quem consome essa substância, mas também impedem os efeitos danosos e indesejáveis aos não consumidores, preservando e protegendo a saúde pública. Da mesma forma, aulas que incluem temas de sexualidade, uso de drogas e DST/AIDS nas grades curriculares. Essas estratégias funcionam para prevenção e promoção da 
saúde da população ${ }^{(8,13)}$.

Em junho de 2009, o ministério lançou o Plano Emergencial de Ampliação de Acesso ao Tratamento e Prevenção em Álcool e outras Drogas (PEAD), direcionado aos 100 maiores municípios brasileiros, com mais de 250 mil habitantes, todas as capitais e sete municípios de fronteira que foram selecionados, totalizando 108 municípios. Essas cidades somam 77,6 milhões de habitantes, que correspondem a $41,2 \%$ da população nacional ${ }^{(14)}$.

O PEAD busca alcançar, prioritariamente, crianças, adolescentes e jovens em situação de grave vulnerabilidade social, por meio das ações de prevenção, promoção e tratamento dos riscos e danos associados ao consumo prejudicial de substâncias psicoativas. Para atingir os objetivos, foram previstos quatro eixos de atuação: ampliação do acesso, qualificação dos profissionais, articulação intra/intersetorial, bem como a promoção da saúde e dos direitos, além do enfrentamento do estigma ${ }^{(14)}$.

Em dezembro de 2009, o Ministério da Saúde lançou a Campanha Nacional de Alerta e Prevenção do Uso de Crack, considerada iniciativa inédita para prevenir o consumo da droga, que é derivada da cocaína e possui alto grau de dependência, com o slogan "nunca experimente o crack" $^{(14)}$.

Associado à Campanha Nacional de Alerta e Prevenção ao Uso de Crack, o Ministério da Saúde divulgou o incentivo financeiro para os projetos chamados Consultórios de Rua, em 50 municípios, com aproximadamente $500 \mathrm{mil}$ habitantes, para oferecerem ações de promoção da saúde, cuidados básicos e redução de danos a pessoas que moram ou estão em situação de rua e são usuárias ou dependentes de álcool e drogas ${ }^{(14)}$.

Desde 2006, o Ministério vem desenvolvendo, em conjunto com pesquisadores da Faculdade de Medicina de Ribeirão Preto, o Programa de ações integradas para a prevenção e atenção ao uso de álcool e outras drogas na comunidade - PAI-PAD, que visa capacitar profissionais da Atenção Básica para o rastreamento de consumo de álcool e utilização de estratégias de intervenção breve - $\operatorname{IBs}^{(14)}$.

Duas outras medidas do Ministério da Saúde consistem na implantação de 60 Casas de Passagem, estruturas destinadas a abrigar usuários de álcool e drogas em situação de risco, e de 70 Pontos de Acolhimento a usuários de crack e outras drogas, ação já adotada em vários países. Os Pontos de Acolhimento são espaços abertos, em centros urbanos com mais de 400 mil habitantes, que recebem pessoas para alimentação, banho e descanso ${ }^{(14)}$.

\section{Discussão}

O movimento de reforma psiquiátrica iniciado com o processo da Reforma Sanitária no Brasil foi, posteriormente, encabeçado pelo Movimento dos Trabalhadores de Saúde Mental, de caráter contra-hegemônico, considerado como dispositivo político no projeto da Reforma Psiquiátrica Brasileira. Assim, emergiram propostas de reformulação do sistema assistencial, que consolidou o pensamento crítico no saber psiquiátrico ${ }^{(15)}$.
Na cultura ocidental, a vontade está associada às condições objetivas, pois se tem duas distinções sociais de subjetividades: rebeldes e conformistas. As subjetividades rebeldes são aquelas que estão sempre a buscar mudanças, não se conformam com a situação presente ${ }^{(15-16)}$. Essas subjetividades transgressivas não se articulam pela via da política, em ação conformista, mas pela promoção da ação rebelde.

Subjetividades rebeldes são capazes de produzir alternativa à hegemonia conservadora e neoconservadora e seus feitos na ampliação da barbárie humano-social ${ }^{(16-17)}$.

No cenário histórico da Reforma Psiquiátrica, os trabalhadores da saúde mental - as subjetividades rebeldes - buscaram no poder hegemônico, representado pelo Ministério da Saúde, a via de acesso para que suas reivindicações e propostas fossem legitimadas. Nesse sentido, as manifestações de trabalhadores e acadêmicos do cenário de atuação/estudo em álcool e outras drogas sensibilizaram o Ministério da Saúde para a mudança do status de muitos desses serviços, do nível II para III, o que minimizará as dificuldades enfrentadas na intervenção dos problemas de saúde, associados ao consumo de substâncias psicoativas, que acometem os usuários e muitas vezes requerem internações breves ${ }^{(14,18)}$.

A desconstrução do paradigma de exclusão da pessoa que tem uma existência- sofrimento e a construção de um novo, que traz a perspectiva de conviver com as diferenças, é processual; ainda mais quando essas figuras humanas excluídas da sociedade não participam do processo de produção e representam ônus à parcela produtiva da população.

Embora as reformas na saúde, educação ou previdência social anunciadas no mundo pós-moderno sejam, na maioria das vezes, para piorar a vida dos cidadãos, há de se considerar que, teoricamente, a Política Nacional para a atenção aos usuários de álcool e outras drogas, do Ministério da Saúde brasileiro, concorre para a emancipação social dessas pessoas ${ }^{(17)}$, porque propõe estratégias que promovem o cuidado, a autonomia, a reabilitação psicossocial e a inclusão social pelo trabalho, uma vez que as pessoas se reconhecem como cidadãs, pelo trabalho.

Os autores utilizam a expressão "teoricamente" porque, embora estejam prescritas na Política estratégias que enfocam a tríade da reabilitação psicossocial: moradia, trabalho e rede social, muitas vezes essas não se materializam no cotidiano dos CAPSad, dada a complexidade dessas questões e as dificuldades de articulação intersetoriais.

Em que pese seja maior a associação da violência com o narcotráfico, estudos revelam que os índices de violência doméstica e urbana, entre as pessoas que consomem substâncias psicoativas, são mais elevados do que entre aquelas que não fazem uso delas, além do maior envolvimento em acidentes de trânsito, estando sob efeito dessas substâncias ${ }^{(19-20-21,22)}$. Assim, as estratégias propostas pela Política Nacional do Ministério da Saúde concorrem para autonomia e emancipação do sujeito social, uma vez que ela se propõe a diminuir a participação dos usuários 
nesse censo epidemiológico.

Aqui, os autores entendem a emancipação do usuário como sendo a sua reabilitação psicossocial. A emancipação do sujeito é processo de resgate da dignidade, da condição de cidadania. Porque pensar em emancipar é pensar na promoção da liberdade, igualdade e solidariedade.

O espaço onde ela acontecerá é a comunidade, porque o papel do Estado é o da regulação social, que o faz contrapor-se ao princípio de emancipação. No entanto, "a comunidade assenta na obrigação política horizontal entre indivíduos ou grupos sociais e na solidariedade que dela decorre, uma solidariedade participativa e concreta, isto é, socialmente contextualizada”. Assim, sob o ponto de vista de emancipação, é possível pensar em outras formas de cidadania, mais assentadas em participação do que em direitos e deveres, não liberais e não estatizantes, mas que viabilizem relação mais equilibrada com a subjetividade ${ }^{(23)}$.

A Sociologia das ausências é um procedimento transgressivo, uma Sociologia insurgente para tentar mostrar que o que não existe é produzido como não existente, como uma alternativa não crível, como uma alternativa descartável, invisível à realidade hegemônica do mundo ${ }^{(10)}$. Existem modos de produzir ausências e o referencial se desenvolve em oposição às seguintes racionalidades: monocultura do saber e do rigor do saber, monocultura do tempo linear, a lógica da classificação social, escala dominante e a lógica da não existência lógica produtivista ${ }^{(22)}$.

Até pouco tempo atrás, a única política pública que se tinha para o uso abusivo de álcool e outras drogas era coercitiva e não se aproximava das questões relacionadas à saúde e à subjetividade da pessoa humana. $\mathrm{O}$ beber compulsivo estava associado ao desvio de conduta moral e era de alçada da justiça. Não que essa percepção inexista na sociedade, mas hoje já se observam discussões acerca das vulnerabilidades sociais relacionadas ao beber pesado e ao uso de outras drogas, consideradas ilícitas, e o quanto esse consumo leva a pessoa à condição de adoecimento e de existência-sofrimento.

Existem, atualmente, duas políticas públicas para as questões relacionadas ao consumo de álcool e outras drogas: uma elaborada pelo Ministério da Saúde, de atenção aos usuários de álcool e outras drogas, em análise neste trabalho, e outra, a aprovada pelo Conselho Nacional Antidrogas da Secretaria Nacional de Políticas sobre Drogas - Senad.

Os autores admitem que a Política Nacional do Ministério da Saúde tem sido construída com a participação de usuários, familiares e trabalhadores, que se expressam no coletivo, como o verificado na IV Conferência de Saúde Mental, realizada em junho/julho de 2010, em Brasília. É exatamente a "participação" de segmentos de interesse da sociedade que lhe confere o caráter contra-hegemônico, de produzir realidades onde imperava o vazio, a inexistência.

Nos serviços de saúde mental, de caráter comunitário, muitos trabalhadores atuam como subjetividades rebeldes e com maior compreensão das demandas dos usuários, conseguem desenvolver um modelo de atenção em saúde, que envolve acolhimento, humanização, vínculo e atenção integral à saúde. Essas práticas são de oposição ao modelo médico e hospitalocêntrico.

Nessa perspectiva, na área de atenção aos usuários, podem emergir outras estratégias contra-hegemônicas que não são de conhecimento da sociedade, porque ainda é um conhecimento não existente, não reconhecido. "A Sociologia das ausências e a Sociologia das emergências produzirão uma enorme quantidade de realidades que não existiam antes"(17). Pela via do saber que emerge do cotidiano, podem-se criar estratégias que responderão às necessidades daqueles que vivem realidades consideradas inexistentes.

A lógica da redução de danos, que começou a ser aplicada no Brasil, na década de 1980, se coloca na contramão do modelo hegemônico da abstinência. Lógica essa que associa tratamento à ausência de consumo, sem considerar a necessidade, a dependência e o direito do sujeito-cidadão.

A redução de danos não exclui a abstinência, como objetivo aos indivíduos dependentes, mas, realisticamente, possibilita às pessoas escolhas mais pragmáticas para limitar o seu consumo. Por outro lado, auxilia as pessoas a se engajarem, motivando-as ao contato com serviços de tratamento, quando se sentirem prontas. A redução de danos opera na lógica da Sociologia das emergências, que defende a ideia de se trabalhar com as possibilidades das pessoas.

Nessa perspectiva, a sucessão de horizontes leva para um estado final e aborda três características modais da existência: realidade, necessidade e possibilidade, sendo a última desprezada pela ciência moderna ${ }^{(10)}$.

A Sociologia das emergências é a investigação das alternativas que se encaixam no horizonte das possibilidades concretas. Essa Sociologia amplia o presente, incorporando a ele todas as possibilidades e expectativas que ele comporta. A ampliação do presente implica na contração do futuro, na medida em que o "ainda não", longe de ser um futuro infinito e vazio, é um futuro concreto, mesmo incerto e sempre em perigo ${ }^{(10)}$.

Sob a racionalidade de uma Sociologia das emergências, cuidar dos usuários de álcool e outras drogas é proceder a uma ampliação simbólica das práticas, saberes e agentes, de maneira a identificar as tendências do futuro, ou seja, o "ainda não", sobre o qual se pode atuar para maximizar a possibilidade de esperança em relação à probabilidade de frustração. Essa ampliação simbólica é, na verdade, uma imaginação sociológica e política que tem duplo objetivo: conhecer melhor as condições de possibilidade de esperança e definir as ações que promoverão a realização dessas condições ${ }^{(10)}$. Assim, as práticas de redução de danos se colocam no "ainda não" dos usuários, torna-se uma possibilidade, uma esperança de ainda conseguirem deixar o consumo. Já na concepção de abstinência, a maioria dos usuários vivencia a imperiosa frustração de inúmeras tentativas malsucedidas.

Da mesma maneira, as campanhas do Ministério da Saúde, de enfrentamento ao crescente consumo de crack, no Brasil, lançam mão de novas estratégias, como os consultórios de rua e casas de passagem; enfim, investem 
em possibilidades que coincidem com o modo de vida dos usuários que, com muita frequência, já se tornaram desafiliados sociais, sem trabalho, moradia e relações sociais. Essas estratégias significam, para eles, uma possibilidade de emancipação social, no plano individual.

Por fim, campanhas preventivas ao consumo de álcool e outras drogas, com ênfase no crack, têm sido veiculadas nos meios de comunicação.

Este trabalho cumpre seu propósito, ao evidenciar que a Política Nacional do Ministério da Saúde para a atenção integral aos usuários de álcool e outras drogas foi elaborada após a grande movimentação de trabalhadores, usuários e familiares, em torno da reivindicação de um tratamento mais digno e social-reabilitativo para aquele que vivencia uma existência-sofrimento decorrente da dependência de substâncias psicoativas.

\section{Considerações Finais}

A atual Política Nacional do Ministério da Saúde brasileiro para a atenção integral dos usuários de álcool e outras drogas observa as prescrições das Conferências de Saúde Mental e da Lei $\mathrm{n}^{\circ} 10.216$, que redirecionou o modelo de atenção, buscando a reabilitação psicossocial das pessoas que sofrem de transtornos mentais e dos usuários de álcool e outras drogas.

A Política Nacional atende às demandas dos usuários, na medida em que implantou os serviços de base comunitária, e, atualmente, investe recursos em novos modelos de serviços e programas, como os consultórios de rua, casa de passagem, PAI-PAD e PEAD, com a expectativa de reabilitar um maior número de pessoas e prevenir que outras pessoas se tornem dependentes.

Na perspectiva do Paradigma Emergente, entendese a reabilitação psicossocial como emancipação social das pessoas com existência-sofrimento. Por sua vez, a emancipação social é produzida a partir da Sociologia das ausências e das emergências, na perspectiva de inclusão social por meio de estratégias e possibilidades que são consideradas pela sociedade como inexistentes, por não serem expressivas em sua escala de valores.

Entende-se, sob essa óptica, que os trabalhadores de saúde mental têm por desafio perceber o presente da vida dos usuários como algo efêmero e que, se não lançarem mão dessas estratégias, hoje, os mesmos continuarão a não ter o que colher no futuro, mantendo a retroalimentação da dependência.

Em suma, se a Política foi concebida a partir das demandas sociais, o que lhe confere legitimidade, o Ministério da Saúde e os trabalhadores em saúde mental devem investir muito, a fim de que ofereçam, no futuro, a assistência que desejam para os usuários e dependentes de álcool e outras drogas. Para que isso ocorra, é necessário que se lance mão de uma assistência mais humana, caracterizada como uma maior sensibilidade para escuta, destituída de preconceitos. Essa assistência poderá ser viabilizada pela mudança de atitudes, busca de conhecimentos, aperfeiçoamento de habilidades e reconhecimento da existência do outro, a partir do que é considerado inexistente.

\section{Agradecimentos}

À Coordenação de Aperfeiçoamento de Pessoal de Nível Superior - Capes, pela Bolsa de Estágio de Doutoramento Sandwich, no Centro de Estudos Sociais da Faculdade de Economia - Universidade de Coimbra Portugal.

\section{Referencias}

1. World Health Organization. Principles of drugs dependence treatment. [Internet] 2008. [acesso 13 maio 2009]. Disponível em: http://www.who.int/substance abuse/publications/principles_drug_dependence_ treatment.pdf

2. Santos ECV dos, Martin D. Cuidadoras de pacientes alcoolistas no município de Santos, SP, Brasil. Rev Bras Enferm. 2009;62(2):194-9.

3. Nicastri R, Ramos SP. Prevenção do uso de drogas. J Bras Dep Quim. 2001;2(1):34-7.

4. Galassi AD, Alvarenga PG, Andrade A G, Couttolenc BF. Custos dos problemas causados pelo abuso do álcool. Rev Psiquiatr Clin. [periódico na Internet]. 2008. [acesso 13 maio 2009]; 35(1):25-30. Disponível em: http://www. scielo.br/pdf/rpc/v35s1/a07v35s1.pdf

5. Minayo MCdeS, Deslandes SF. A complexidade das relações entre drogas, álcool e violência. Cad Saúde Pública. [periódico na Internet]. 1998. [acesso 05 maio 2011]; 14(1):35-42, jan-mar 1998. Disponível em: http:// www.scielo.br/pdf/csp/v14n1/0123.pdf

6. Ramos SP. Muito além do alcoolismo. In: É bom se preparar que a conversa vai ser muito forte. Porto Alegre. SIMERS. [Internet] 2005. [acesso $24 \mathrm{fev}$ 2007]; 68 p. Disponível em: http://www.simers.org.br/livro_forum alcool_2005.pdf.

7. Ministério da Saúde (BR). A política do Ministério da Saúde para atenção integral a usuários de álcool e outras drogas. Brasília: Ministério da Saúde; 2004. Série E. Legislação de Saúde.

8. Souza de J, Kantorski LP. Smad. Rev Eletronica Saúde Mental, Álcool e Drog. [periódico na Internet]. 2007 [acesso 5 maio 2011]: 3(2)art.2. Disponível em: http:// www2.eerp.usp.br/resmad/artigos/2007v3n2a02.pdf

9. Neves JL. Pesquisa qualitativa - características, usos e possibilidades. Cad Pesqui Admin. [periódico na Internet]. 1996. [acesso 15 maio 2009]:1(3):5. Disponível em: http:// www.ead.fea.usp.br/cad-pesq/arquivos/c03-art06.pdf

10. Santos B de S. Uma sociologia das ausências e uma sociologia das emergências. In: A gramática do tempo: para uma nova cultura política. [para um novo senso comum. A ciência, o direito e a política na transição paradigmática]. Porto: Edições Afrontamento; 2006. p. 87-124.

11. Inverso. Legislação Brasileira. [acesso 13 maio 2009]. Disponível em: http://www.inverso.org.br/blob/194.pdf 12. Pinho PH, Oliveira MA, Almeida MM. A reabilitação psicossocial na atenção aos transtornos associados ao consumo de álcool e outras drogas. Rev Psiquiatr. Clin [periódico na Internet]. 2008 [acesso 13 maio 2009]; 35(1):82-8. Disponível em: http://www.scielo.br/pdf/rpc/ v35s1/a17v35s1.pdf 
13. Governo do Estado de São Paulo (BR). Lei antifumo $\mathrm{n}^{\mathrm{o}} 13.541$, de 07 de maio de 2009 [acesso 15 julho 2010]. Disponível em: http://www.leiantifumo.sp.gov.br/ 14.. Ministério da Saúde (BR). Ministério da Saúde irá dobrar leitos de internação para usuários de crack e outras drogas. [acesso 16 maio 20011]. Disponível em: http:/portal.saude.gov.br/portal/aplicacoes/noticias/ default.cfm?pg=dspDetalheNoticia\&id_area $=124 \& \mathrm{CO}_{-}$ NOTICIA $=11327$

15. Silva AT de MC da, Barros S, Oliveira MAF de. Políticas de saúde e de saúde mental no Brasil: a exclusão/ inclusão social como intenção e gesto. Rev Esc Enferm USP. 2002;36(1): 4-9.

16. Soares RL. Ausências e emergências: produção do conhecimento e transformação social. MATRIZes [Internet] 2002. [acesso 23 out 2008];1(1):231-5. Disponível em: http://revcom.portcom.intercom.org.br/ index.php/MATRIZes/article/view/4001

17. Santos B de S. Renovar a Teoria Crítica e a emancipação social. Rio de Janeiro: Boitempo Editorial; 2007. 126 p.

18. Pereira MO, Oliveira MAF de. Análise da Política do Minstério da Saúde do Brasil para a atenção integral dos usuários de álcool e outras drogas. [tese]. São Paulo: Escola de Enfermagem de São Paulo da Universidade de São Paulo; 2009.

19. Fonseca AM, Galduroz JCF, Tondowski CS, Noto AR. Padrões de violência domiciliar associada ao uso de álcool no Brasil. Rev Saúde Pública. [periodico na Internet] 2009. [acesso 17 mai 2011];43(5):743-49. Disponível em: http://www.scielo.br/pdf/rsp/v43n5/24.pdf

20. Heim J, Andrade AGde. Efeitos do uso do álcool e das drogas ilícitas no comportamento de adolescentes de risco: uma revisão das publicações científicas entre 1997 e 2007. Rev Psiquiatr Clín. [periodico na Internet] 2008. [acesso 17 mai 2011]; 35(1):61-4. Disponível em: http:// www.scielo.br/pdf/rpc/v35s1/a13v35s1.pdf

21. Barata RB, Ribeiro M CSdeA, Sordi Mde. Desigualdades sociais e homicídios na cidade de São Paulo, 1998. Rev Bras Epidemiol. [periodico na Internet] 2008. [acesso 17 mai 2011]; 11(1):3-13. Disponível em: http://www.scielo.br/pdf/rbepid/v11n1/01.pdf

22. Abreu AMM, Lima JMBde, Matos LN, Pillon SC. Uso de álcool em vítimas de acidentes de trânsito: estudo do nível de alcoolemia. Rev. Latino-Am. Enfermagem. [periodico na Internet] 2010. [acesso 17 mai 2011];18:51320. Disponível em: http://www.scielo.br/pdf/rlae/v18nspe/ a05v18nspe.pdf
23. Santos B de S. Subjetividade, cidadania e emancipação. In: Santos B de S. Pela Mão de Alice: o social e o político na pós-modernidade. 5 ed. Porto: Edições Afrontamento; 1996. p. 203-41.

\section{Como citar este artigo:}

Pereira MO, Vargas D, Oliveira MAF. Reflexão acerca da política do ministério da saúde brasileiro para a atenção aos usuários de álcool e outras drogas sob a óptica da Sociologia das ausências e das emergências. SMAD, Rev. Eletrônica Saúde Mental Álcool Drog. (Ed. port.). jan.-abr. 2012 [acesso:

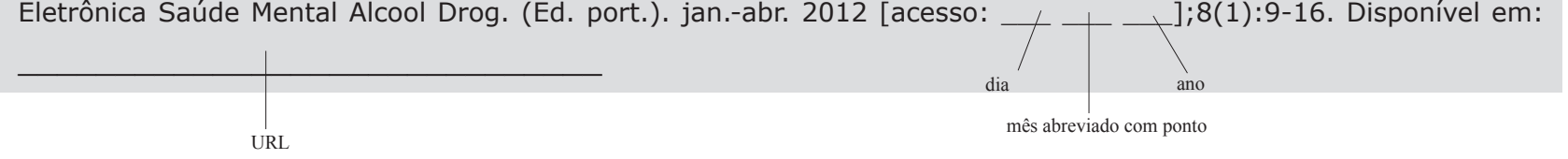

